\title{
Successful control of an outbreak of colonization by Klebsiella pneumoniae carbapenemase-producing $K$. pneumoniae sequence type 258 in a neonatal intensive care unit, Italy
}

\author{
M. Giuffrè ${ }^{a}$, C. Bonura ${ }^{a}$, D.M. Geraci ${ }^{\text {a, b }}$, L. Saporito ${ }^{a}$, R. Catalano ${ }^{a, c}$, \\ S. Di Noto ${ }^{a, d}$, F. Nociforo ${ }^{a, d}$, G. Corsello ${ }^{a}$, C. Mammina ${ }^{a, *}$ \\ a Department of Sciences for Health Promotion and Mother-Child Care 'G. D'Alessandro', University of Palermo, Palermo, Italy \\ ${ }^{\mathrm{b}}$ PhD School in Food and Human Nutrition, University of Palermo, Palermo, Italy \\ ' Postgraduate Specialty School in Preventive Medicine, University of Palermo, Palermo, Italy \\ ${ }^{\mathrm{d}}$ Postgraduate Specialty School in Pediatrics, University of Palermo, Palermo, Italy
}

\section{A R T I C L E I N F O}

\section{Article history:}

Received 8 April 2013

Accepted 8 August 2013

Available online 8 September

2013

\section{Keywords:}

Colonization

Infection control

KPC-Klebsiella pneumoniae

ST258

Multidrug resistance

Neonatal intensive care unit

\section{$S \cup M M A R$}

This article reports an outbreak of colonization by Klebsiella pneumoniae carbapenemaseproducing $K$. pneumoniae (KPC-Kp) sequence type (ST) 258 in a neonatal intensive care unit (NICU) in Palermo, Italy. KPC-Kp ST258 was detected by an active surveillance culture programme. Between 18th September and 14th November 2012, KPC-Kp was isolated from 10 out of 54 neonates admitted in the outbreak period. No cases of infection were recorded. Male sex was associated with colonization, whereas administration of ampicillinsulbactam plus gentamicin was protective. Infection control interventions interrupted the spread of KPC-Kp without the need to close the NICU to new admissions.

(c) 2013 The Healthcare Infection Society. Published by Elsevier Ltd. All rights reserved.

\section{Introduction}

Over the last decade resistance to carbapenems due to the production of Klebsiella pneumoniae carbapenemases (KPCs) has spread worldwide. ${ }^{1}$ KPCs are often associated with

\footnotetext{
* Corresponding author. Address: Department of Sciences for Health Promotion and Mother-Child Care 'G. D'Alessandro', University of Palermo, Via del Vespro 133, I-90127 Palermo, Italy. Tel.: +39 0916553623; fax: +390916553641.

E-mail address: caterina.mammina@unipa.it (C. Mammina).
}

internationally successful $K$. pneumoniae clones, such as sequence type (ST) 258, which are endemic in many geographic areas, including Italy. ${ }^{1}$

Gram-negative bacteria cause $\sim 20-40 \%$ of all late-onset sepsis in the neonatal intensive care units (NICUs) and are frequently associated with adverse clinical outcomes. ${ }^{2,3}$ Colonization with multidrug-resistant (MDR) Gram-negative organisms is also, therefore, of great concern, due to the possibility of infection. ${ }^{4}$ Additionally, colonized infants can sustain unrecognized chains of transmission and create long-term reservoirs of MDR bacteria and drug resistance genes. ${ }^{3}$ 
We report an outbreak of colonization by KPC-Kp ST258 in the NICU of the University Hospital of Palermo, Italy, and describe the measures that allowed for its successful control.

\section{Methods}

\section{Setting}

The tertiary-level NICU of the University Hospital Azienda Ospedaliero-Universitaria Policlinico 'P. Giaccone', Palermo, Italy, admits about 250 infants annually. Because it is associated with the regional reference centre for genetic diseases, the NICU has a high prevalence of neonates with malformations $(\sim 20 \%)$, as well as of admissions referred from elsewhere ( $\sim 35 \%)$. A further $20 \%$ of patients have complex conditions requiring specialist medical or surgical care. The NICU includes one intensive care room consisting of eight cot spaces and one intermediate care room with eight further cot spaces. The average nurse:patient ratio is $1: 3$ and 1:4 in the two sections, respectively.

Since June 2009, a routine surveillance protocol is in place which includes nasal and rectal swabs obtained on a weekly basis to monitor the prevalence of colonization with meticillinresistant Staphylococcus aureus, multidrug-resistant (MDR) Gram-negatives and glycopeptide-resistant enterococci.

All infants admitted to the NICU between 4th September and 21st November 2012 were included in the study. Healthcare-associated infections were defined using standard Centers for Disease Control and Prevention (CDC) definitions adapted to the neonatal clinical features. ${ }^{5}$ Colonization was defined as a positive culture of KPC-Kp from at least one rectal swab collected during the NICU stay.

The study protocol was approved by the Ethics Committee of the Azienda Ospedaliero-Universitaria Policlinico
'P. Giaccone', Palermo, Italy. Written informed consent was obtained from the parents of each patient.

\section{Case-control study}

A case-control study was performed to identify possible risk factors for KPC-Kp colonization. Controls were those infants admitted between 4th September and 21st November and who had negative surveillance cultures. Risk factors at admission and during NICU stay were assessed. For cases, risk factors during NICU stay were assessed for the interval between admission and the first positive culture, whereas for controls, risk factors were assessed for the interval between admission and discharge. Univariate analysis was carried out by the Epi Info software (version 7; CDC, Atlanta, GA, USA). Tests of significance were performed with chi-square test or Student's $t$ test where appropriate. Stepwise logistic regression analysis of variables found to be significant in the univariate analysis $(P<0.10)$ was performed using StatPlus 2009 software. All significance tests were two-tailed, and $P<0.05$ was considered significant.

\section{Microbiological investigations}

Intestinal colonization by antibiotic-resistant Gram negatives was assessed by culturing overnight broth-enriched rectal swabs onto MacConkey agar plates with disks of meropenem $(10 \mu \mathrm{g})$ and ceftazidime $(30 \mu \mathrm{g}) .^{6}$ Colonies growing within antibiotic inhibition zones were subcultured, biochemically identified and submitted for antimicrobial susceptibility testing and ESBL detection according with the European Committee on Antimicrobial Susceptibility Testing (EUCAST) guidelines (http://www.eucast.org/clinical_breakpoints/). Phenotypic screening for carbapenemases or overexpression of

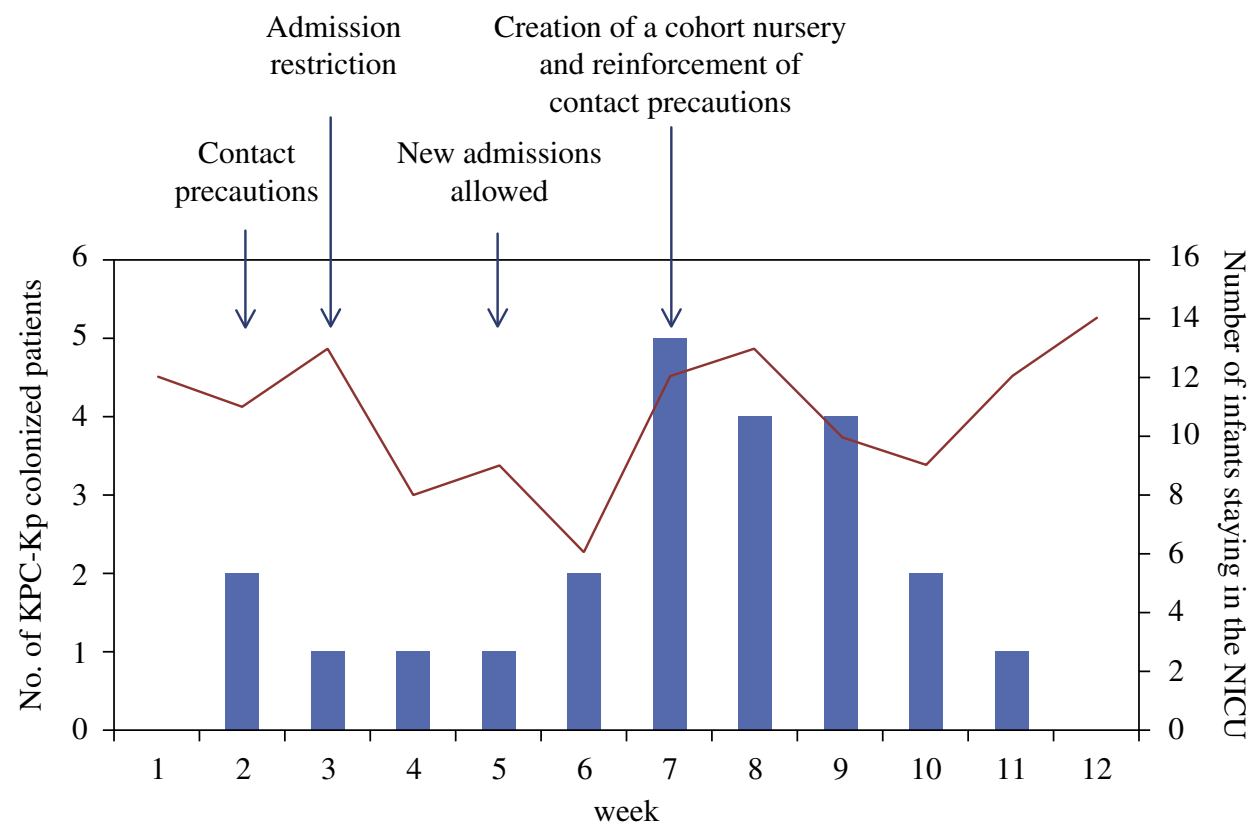

Figure 1. Epidemic curve showing Klebsiella pneumoniae carbapenemase-producing K. pneumoniae (KPC-Kp) sequence type (ST) 258 colonizations during the outbreak period and timing of the infection control interventions. 
$\mathrm{AmpC}$ in combination with porin loss was performed by a commercial test (Rosco Diagnostica, Taastrup, Denmark). $E$. coli ATCC 25922 was used as the control strain. $\beta$-Lactamase genes were identified by polymerase chain reaction amplification and sequencing. Multilocus sequence typing (MLST) was performed and sequence types were determined using the K. pneumoniae MLST database: http://www.pasteur.fr/ recherche/genopole/PF8/mlst/Kpneumoniae.html.

During the outbreak period, repeated surveys of environmental contamination by carbapenem-resistant Enterobacteriaceae were carried out.

\section{Results}

\section{Outbreak description and infection control interventions}

Between 18th September and 14th November 2012, KPC-Kp was isolated from 10 neonates. The epidemic curve is illustrated in Figure 1. The index cases were two neonates born elsewhere, who had been admitted to the NICU on 6th and 11 th September, respectively, and tested positive for KPC-Kp on 18th September. They had been transferred from another general hospital and from a private maternity clinic, respectively, where no cases of infection by KPC-Kp had been reported. Infection prevention and control measures were implemented promptly. The two index cases were isolated in incubators and contact precautions were initiated. Extensive environmental microbiological investigations were performed to identify possible reservoirs or sources, but were unsuccessful. The NICU environment was thoroughly sanitized and instructions regarding reinforcement of hand hygiene were given. The two neonates remained under contact isolation precautions until discharge. A temporary restriction of admissions was also decided upon (Figure 1). However, on 16th October, a third neonate, who had been in NICU since June because of a complex malformation syndrome, was found to be colonized with KPC-Kp. Thereafter, between 23th October and 14th November, seven further neonates acquired KPC-Kp colonization. On 23th October, additional control interventions were put in place (Figure 1): a separate intermediate care room was identified as a cohort room and colonized infants were transferred there, as soon as their clinical conditions were compatible with a lower level of care, and were kept under contact precautions until discharge. This procedure was not feasible with the third patient, because of his severe malformation which required stay in the intensive care room, where isolation was performed by placing him in incubator at one end of the room and keeping the adjacent cot empty. The use of dedicated patient equipment, but not designated staff, was undertaken. Standard infection control procedures were reinforced with special attention to hand hygiene upon entry in the NICU, use of alcohol-based hand sanitizers between patient contacts and cleaning and sanitization of the environment. Weekly educational meetings in the form of short talks were arranged, and involved all healthcare workers and alerted them to the outbreak course (Figure 1). On 21st November, only the third colonized neonate was still proven to carry KPC-Kp. He died from surgical complications on 21st November. There were no further cases of colonization or infection by carbapenem-resistant Enterobacteriaceae in the NICU during the following seven months.

Table I

Univariate and multivariate analysis of risk factors for acquisition of colonization by Klebsiella pneumoniae carbapenemase-producing K. pneumoniae ST258 in patients in the neonatal intensive care unit, September to November 2012

\begin{tabular}{|c|c|c|c|c|c|c|}
\hline \multirow[t]{2}{*}{ Variable } & \multirow[t]{2}{*}{ Cases $(N=10)$} & \multirow{2}{*}{ Controls $(N=44)$} & \multicolumn{2}{|c|}{ Univariate analysis } & \multicolumn{2}{|c|}{ Multivariate analysis } \\
\hline & & & OR $(95 \% \mathrm{Cl})$ & $P$-value & OR $(95 \% \mathrm{Cl})$ & $P$-value \\
\hline Gender (male) ${ }^{a}$ & $9(90.0)$ & $19(43.2)$ & $10.42(1.21-89.94)$ & 0.007 & $10.53(1.15-96.01)$ & 0.02 \\
\hline Birth weight $(\mathrm{g})^{\mathrm{b}}$ & $2639.0(816.8)$ & $2764.3(747.7)$ & & 0.64 & & \\
\hline Gestational age (weeks) ${ }^{\mathrm{b}}$ & $36.4(1.7)$ & $36.7(3.3)$ & & 0.80 & & \\
\hline Caesarean section $^{\mathrm{a}}$ & $9(90.0)$ & $22(50.0)$ & $1.41(1.12-1.76)$ & 0.02 & $1.68(0.86-1.98)$ & 0.09 \\
\hline Twin birth ${ }^{a}$ & 0 & $1(2.3)$ & & & & \\
\hline Malformation $^{a}$ & $2(20.0)$ & $10(22.7)$ & $0.83(0.15-4.66)$ & 0.44 & & \\
\hline Inborn $^{a}$ & $3(30.0)$ & $24(54.5)$ & $0.30(0.07-1.34)$ & 0.07 & $0.29(0.07-1.43)$ & 0.13 \\
\hline Central vascular catheter ${ }^{a}$ & $3(30.0)$ & $20(45.4)$ & $0.45(0.10-1.98)$ & 0.16 & & \\
\hline Endotracheal tube $^{a}$ & $3(30.0)$ & $14(31.8)$ & $0.83(0.18-3.70)$ & 0.42 & & \\
\hline $\begin{array}{l}\text { Nasal continuous positive } \\
\text { airway pressure }\end{array}$ & $4(40.0)$ & 7 (15.9) & $3.24(0.72-14.57)$ & 0.08 & & \\
\hline Nasogastric tube $e^{a}$ & $5(50.0)$ & $19(43.2)$ & $1.16(0.29-4.62)$ & 0.42 & & \\
\hline Parenteral nutrition $^{a}$ & $9(90.0)$ & $34(77.3)$ & $1.85(0.20-17.07)$ & 0.33 & & \\
\hline Ampicillin-sulbactam/gentamicin ${ }^{a}$ & $4(40.0)$ & $31(70.4)$ & $0.21(0.05-0.92)$ & 0.02 & $0.20(0.03-0.97)$ & 0.004 \\
\hline Ampicillin-sulbactam (days) ${ }^{\mathrm{b}}$ & $6.1(11.3)$ & $7.5(7.4)$ & & 0.64 & & \\
\hline Gentamicin (days) ${ }^{\mathrm{b}}$ & $4.1(6.3)$ & $5.8(7.0)$ & & 0.49 & & \\
\hline Length of stay ${ }^{c}$ & $12(4-135)$ & $12(4-118)$ & & 0.75 & & \\
\hline All-cause mortality & $1(10.0)$ & $5(11.4)$ & $1.2(0.13-12.07)$ & 0.46 & & \\
\hline
\end{tabular}

$\mathrm{OR}$, odds ratio; $\mathrm{Cl}$, confidence interval.

a Frequency (\%).

b Mean (SD).

c Median (range). 


\section{Case-control study}

Forty-four neonates with negative surveillance cultures were included as controls in the study. Male gender was significantly associated with KPC-Kp colonization, whereas administration of ampicillin-sulbactam and gentamicin was negatively associated by both univariate and multivariate analysis (Table I). No significant difference was observed in allcause mortality between cases and controls.

\section{Microbiological findings}

The KPC-Kp isolates from the 10 neonates were resistant to imipenem (MICs $\geq 16 \mu \mathrm{g} / \mathrm{mL}$ ), meropenem (MICs $2 \mu \mathrm{g} / \mathrm{mL}$ ) and ertapenem (MICs $\geq 8 \mu \mathrm{g} / \mathrm{mL}$ ). They were also resistant to aminoglycosides, except for gentamicin (MICs $4 \mu \mathrm{g} / \mathrm{mL}$ ), fluoroquinolones and trimethoprim-sulfamethoxazole. They were susceptible to colistin (MICs $\leq 0.5 \mu \mathrm{g} / \mathrm{mL}$ ) and tigecycline (MICs $\leq 1 \mu \mathrm{g} / \mathrm{mL}$ ). All isolates were positive for the presence of the KPC-3 sequence. The KPC-Kp strain was attributed with sequence type (ST)258.

\section{Discussion}

KPC-Kp infections are increasingly frequent worldwide, representing a major clinical and public health threat. Italy ranks third among the European countries, after Greece and Cyprus, by prevalence of KPC-Kp infections. ${ }^{7}$ Previous reports have described the epidemic spread of KPC-Kp ST258 in different healthcare settings in Sicily. ${ }^{8,9}$ However, to the best of our knowledge, this is the first report of an outbreak of colonization by KPC-Kp in NICU. Interestingly, the emergence of the outbreak strain coincided with the admission of two neonates referred from elsewhere, but the most prominent role as the source of transmission was likely played by the third long-stay patient with malformation. Indeed, persistent carriage is especially challenging because of the need for strict adherence to contact precautions over extended periods of time.

The outbreak was interrupted, without NICU closure, after about two months, even though the source of the KPC-Kp strains was not identified. Environmental sampling yielded no carbapenem-resistant bacteria, but alternative sources of KPC$\mathrm{Kp}$ could not be ruled out, including the possible carriage by healthcare workers or parents. Furthermore, frequently reported risk factors in NICU patients, such as low birth weight, prematurity, length of stay, use of invasive devices and prolonged receipt of antibiotics, were not identified in our study.

There is no universal agreement about the effectiveness of interventions, such as active surveillance and isolation/ cohorting, in controlling the spread of MDR Gram-negative organisms in NICU. However, in Israel four-year active continuous surveillance combined with contact precautions has been associated with a significant reduction of acquisition of extended-spectrum beta-lactamase (ESBL)-Kp in NICU, compared with no concurrent decrease in the same hospital as a whole. ${ }^{10}$ Moreover, in Germany weekly screening for MDR pathogens has been recently recommended in NICUs not only to obtain a more reliable picture of local epidemiology, but also to allow for interventions on colonized patients aimed at controlling nosocomial transmission and to adjust the choice of the empirical first-line treatment. ${ }^{3}$ In our experience active surveillance cultures allowed the infection prevention and control interventions to be initiated in the absence of clinical infection. This can be especially helpful in NICUs located in areas where carbapenem-resistant $K$. pneumoniae are endemic.

\section{Conflict of interest statement \\ None declared.}

Funding sources

This work was supported by institutional resources.

\section{References}

1. Woodford N, Turton JF, Livermore DM. Multiresistant Gramnegative bacteria: the role of high-risk clones in the dissemination of antibiotic resistance. FEMS Microbiol Rev 2011;35:736-755.

2. Gastmeier P, Loui A, Stamm-Balderjahn S, et al. Outbreaks in neonatal intensive care units: they are not like others. Am J Infect Control 2007;35:172-176.

3. Simon A, Tenenbaum T. Surveillance of multidrug-resistant Gramnegative pathogens in high risk neonates - does it make a difference? Pediatr Infect Dis J 2013;32:407-409.

4. Smith A, Saiman L, Zhou J, Della-Latta P, Jia H, Graham PL. Concordance of gastrointestinal tract colonization and subsequent bloodstream infections with Gram-negative bacilli in very low birth weight infants in the neonatal intensive care unit. Pediatr Infect Dis J 2010;29:831-835.

5. Garner JS, Jarvis WR, Emori TG, Horan TC, Hughes JM. CDC definitions for nosocomial infections, 1988. Am J Infect Control 1988; 16:128-140.

6. Giani T, Tascini C, Arena F, et al. Rapid detection of intestinal carriage of Klebsiella pneumoniae producing KPC carbapenemase during an outbreak. J Hosp Infect 2012;81:119-122.

7. European Centre for Disease Prevention and Control. Antimicrobial resistance surveillance in Europe 2011. Annual Report of the European Antimicrobial Resistance Surveillance Network (EARSNet). Stockholm: ECDC; 2012.

8. Agodi A, Voulgari E, Barchitta M, et al. Containment of an outbreak of KPC-3-producing Klebsiella pneumoniae in Italy. J Clin Microbiol 2011;49:3986-3989.

9. Mammina C, Palma DM, Bonura C, et al. Outbreak of infection with Klebsiella pneumoniae sequence type 258 producing Klebsiella pneumoniae carbapenemase 3 in an intensive care unit in Italy. J Clin Microbiol 2010;48:1506-1507.

10. Benenson S, Levin PD, Block C, et al. Continuous surveillance to reduce extended-spectrum $\beta$-lactamase Klebsiella pneumoniae colonization in the neonatal intensive care unit. Neonatology 2013;103:155-160. 